\title{
Non-Uniform Diffraction Pattern of 2D-Cross-Grating ---Rotating Grating Around Three Axes
}

This paper was downloaded from TechRxiv (https://www.techrxiv.org).

\section{LICENSE}

CC BY 4.0

SUBMISSION DATE / POSTED DATE

$15-02-2022$ / 23-02-2022

\section{CITATION}

peng, hui (2022): Non-Uniform Diffraction Pattern of 2D-Cross-Grating ---Rotating Grating Around Three Axes. TechRxiv. Preprint. https://doi.org/10.36227/techrxiv.19172846.v1

$\mathrm{DOI}$

10.36227/techrxiv.19172846.v1 


\title{
Non-Uniform Diffraction Pattern of 2D-Cross-Grating ---Rotating Grating Around Three Axes (4)
}

\author{
Hui Peng
}

\author{
Email: davidpeng1749@gmail.com
}

\begin{abstract}
The orientation-dependence of the diffraction patterns of the 1D-grating experiments have been systematically studied by utilizing the 3-axis-rotation apparatus. It is shown that the spacings between adjacent diffraction orders are non-uniform. Namely, from "one end" of the pattern to "other end", the spacings between adjacent diffraction orders gradually increase. In this article, we systematically study the orientation-dependence of the diffraction patterns of the 2D-cross-grating. The experiments show that the diffraction patterns are non-uniform as that of the 1D-grating experiments.
\end{abstract}
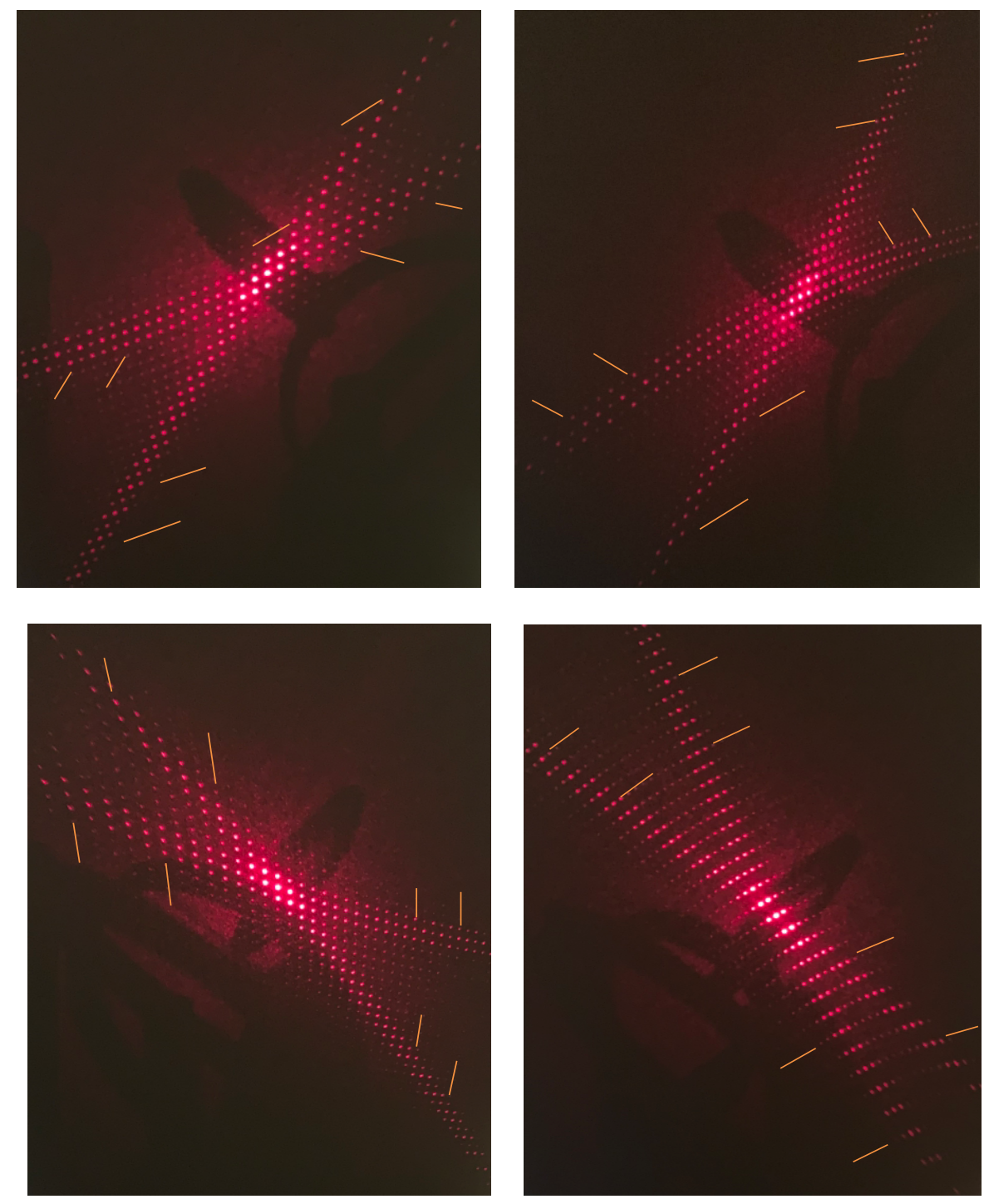

This extraordinary phenomenon challenges the existing theoretical description of the grating experiments. The above observed phenomena provide the comprehensive phenomena to theoretical 
study of the grating experiments. We suggest that the complete mathematical model should contain three rotation angles as parameters and should be able to describe the phenomena of the non-uniformspacing, curved, expanded, inclined and mirror-symmetric diffraction patterns consistently.

Keywords: grating experiment, cross-grating experiments, diffraction pattern, normal incident light beam, oblique incident light beam, orientation-dependence, non-uniform diffraction pattern, curved diffraction pattern, expanded diffraction pattern, inclined diffraction pattern, mirror-symmetric pattern

\section{Introduction}

The orientation-dependence, for certain orientations, of the interference patterns of the 1D-double slit and 2D-cross-double slit experiments have been studied experimentally [1-4]. The universal phenomena of the curved, expanded and inclined patterns were observed.

Recently, we have introduced the 3-axis-rotation apparatus, by which, the orientation-dependence of the diffraction patterns of the 1D-grating experiments have been studied [5-7]. The simultaneously non-uniform spacing, curved, expanded, inclined and mirror-symmetric diffraction patterns (hereafter denoted as "pattern") emerged in the 1D-grating experiments. Indeed, the new phenomena show that the characteristics of the patterns depend on the orientations of the grating.

In this article, we study the orientation-dependence of the patterns of the 2D-cross-grating by utilizing the 3 -axis-rotation apparatus. The $2 \mathrm{D}$-cross-grating is rotated around three axes sequentially and respectively. The study of the patterns is achieved intuitively. We observed the phenomena that, the spacings between adjacent diffraction orders are non-uniform.

The phenomena challenge both the standard theory of the grating for the normal incident light beam and the theories of the grating for the oblique incident light beam [8-11].

The novel phenomena of the continuously non-uniform spacing, curved, expanded, inclined, and mirror-symmetric patterns provide the comprehensive phenomena to theoretically study the grating experiments. We suggest that a complete mathematical model should contains three rotation angles as parameters and should be able to describe all phenomena of the orientation-dependence of the patterns consistently.

\section{Three-Axis-Rotation Apparatus and Associated Coordinate System}

The 3-axis-rotation apparatus has been proposed (Figure 01) [5]. Figure 01 shows the experimental setup and the coordinate. The horizontal slits S1 and the vertical slits S2 create the vertical pattern and horizontal pattern respectively, referred as branch-patterns. In the previous articles [7], the 1D-grating has been rotated around 3 axes. 


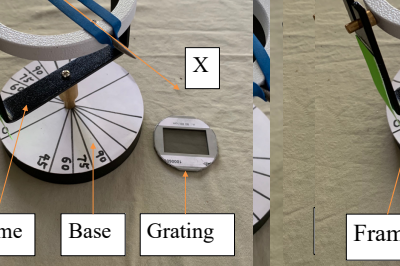

(a)
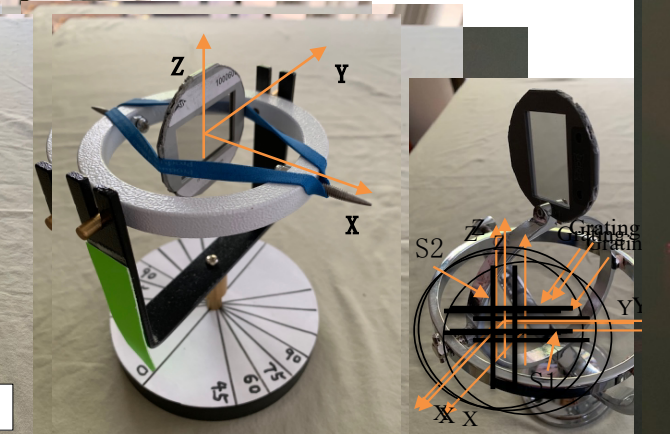

(b)

Figure 01. Experimental setup (a); the 3-axis-rotation apparatus and 2D-cross grating (b):

the $2 \mathrm{D}$-cross-grating can be rotated around $\mathrm{X}$-axis, $\mathrm{Y}$-axis and $\mathrm{Z}$-axis

In this article, we perform four experiments by rotating the 2D-cross-grating around 3 axes.

Namely, rotating the 2D-cross-grating clockwise $(\mathrm{CW})$ and counterclockwise $(\mathrm{CCW})$ around $\mathrm{X}$ axis, $\mathrm{CW}$ and $\mathrm{CCW}$ around $\mathrm{Y}$ axis, and $\mathrm{CW}$ and $\mathrm{CCW}$ around $\mathrm{Z}$ axis respectively and sequentially.

Direction of Rotation: to define the direction of the CW and CCW rotation, we introduce the righthand rule that states that the thumb of the right hand is pointed in the direction of the axis, the CCW rotation is given by the curl of the fingers (Figure 02).

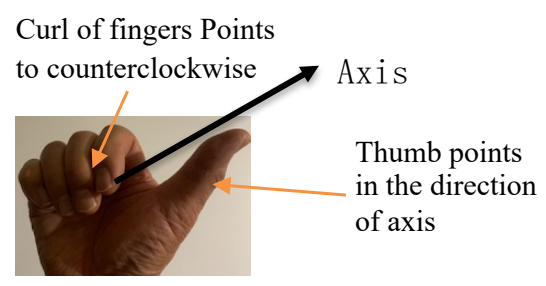

Figure 02. Right-hand rule for determining direction of rotation

CW vs. CCW rotation of the 2D-cross-grating: when rotating $\mathrm{CW}$ and $\mathrm{CCW}$ the $2 \mathrm{D}$-cross-grating around the $\mathrm{X}$-axis by the same angle, the diffraction pattern due to the $\mathrm{CW}$ rotation is the same as that due to the $\mathrm{CCW}$ rotation. Thus, we perform the experiments only with the $\mathrm{CCW}$ rotation around the $\mathrm{X}$ axis. Experiments start with rotating $\mathrm{CCW}$ the $2 \mathrm{D}$-cross-grating around $\mathrm{X}$ axis by $45^{\circ}$ (Figure $03 \mathrm{a}$ ).

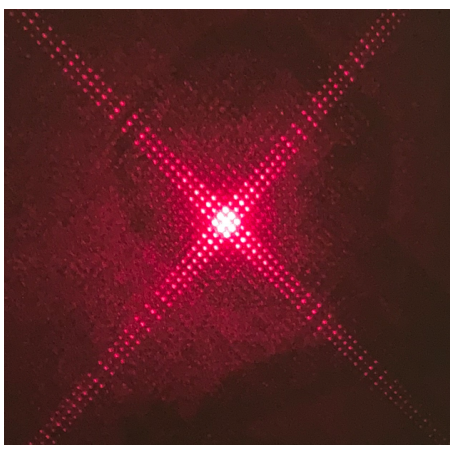

(a)

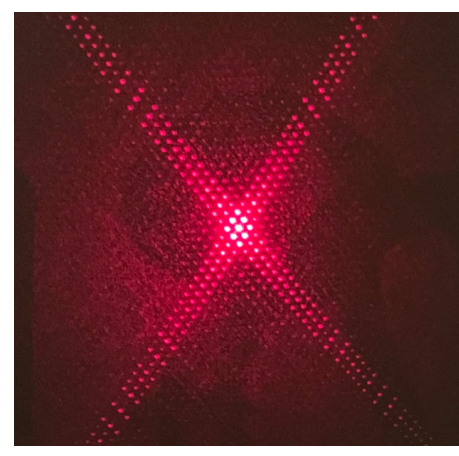

(b)

Figure 03. Patterns; (a) 2D-cross-grating rotating CCW around $\mathrm{X}$ axis by $45^{\circ}$; (B) 2D-cross-grating rotating $\mathrm{CCW}$ around $\mathrm{X}$ axis by $45^{\circ}$ and, then rotating $\mathrm{CW}$ around $\mathrm{Y}$ axis by $45^{\circ}$ 
Figure 03a shows that the first rotation (around the $\mathrm{X}$ axis) of the apparatus causes the pattern rotating, and the characteristics of the pattern have no change. Figure 03b shows that the second rotation (CW around the $\mathrm{Y}$ axis) causes that each branch-pattern expanded, curved, and inclined towards the vertical axis.

In Experiment-1 to -4, we use the specific discrete angles for rotating around each axis, one can rotate the 2D-cross-grating either with any discrete angles or continuously rotating the 2D-crossgrating around any axis.

\section{Experiments: Rotating 1D-grating Around 3 Axes}

\subsection{Rotating 2D-Cross-Grating CCW Around X Axis by $45^{\circ}, \mathrm{CW}$ Around Y Axis by $45^{\circ}$, Then} Rotating $\mathrm{CW}$ and $\mathrm{CCW}$ Around $\mathrm{Z}$ axis

From the orientation of the 2D-cross-grating (Figure 03b), performing the following two Experiments, respectively: rotating the 2D-cross-grating $\mathrm{CW}$ and $\mathrm{CCW}$ around $\mathrm{Z}$ axis respectively. Experiment-1: Rotating the grating: $\mathrm{CCW} X\left(45^{0}\right)+\mathrm{CW} \mathrm{Y}\left(45^{\circ}\right)+\mathrm{CW} \mathrm{Z}\left(\mathbf{7 0}^{0}\right)$

Rotating the grating $\mathrm{CCW}$ around $\mathrm{X}$ axis by $45^{\circ}, \mathrm{CW}$ around $\mathrm{Y}$ axis by $45^{\circ}$ and, then, $\mathrm{CW}$ around $\mathrm{Z}$ axis by $70^{0}$ (Figure 1$)$.

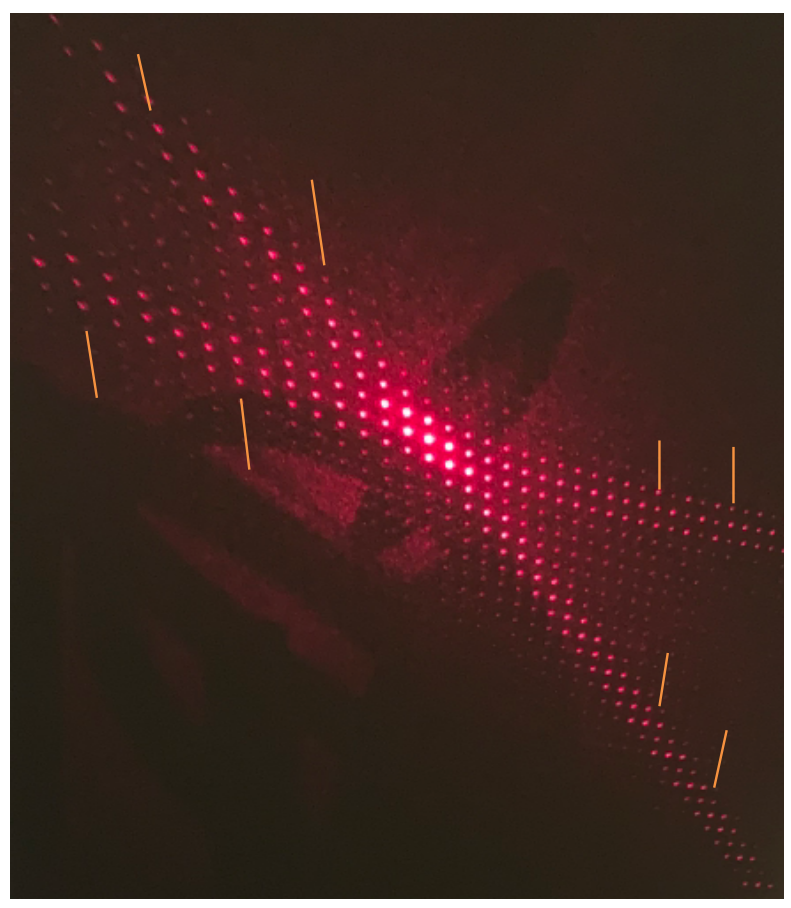

Figure 1. Patterns: rotating 2D-cross-grating CCW around $\mathrm{X}$ axis by $45^{\circ}$, then

$\mathrm{CW}$ around $\mathrm{Y}$ axis by $45^{\circ}$, and $\mathrm{CW}$ around $\mathrm{Z}$ axis by $70^{\circ}$

Figure 1 shows the pattern of the 2D-cross-grating after rotating around 3 axes. 
Observation: The comparison of Figure $03 \mathrm{~b}$ and Figure 1 shows that the third rotation (around the $\mathrm{Z}$ axis) causes that each branch of the patterns expanded more, curved more, and inclined more towards the horizontal axis. The pattern as a whole rotates $\mathrm{CCW}$.

The most significant novel phenomenon of Experiment-1 is that each branch of the pattern is expanded non-uniformly. More specifically, the expansion of the spacing between the adjacent diffraction orders of each branch of the pattern increase gradually from the bottom-portion to the top portion of the pattern. To show the expansion, we use the short-orange-lines that shows the distances between 5 diffraction orders on each branch of the pattern.

\section{Experiment-2: Rotating the grating: $\mathrm{CCW} X\left(4^{\circ}\right)+\mathrm{CW} \mathrm{Y}\left(45^{\circ}\right)+\mathrm{CCW} \mathrm{Z}\left(7^{0}\right)$}

Rotating the grating $\mathrm{CCW}$ around $\mathrm{X}$ axis by $45^{\circ}, \mathrm{CW}$ around $\mathrm{Y}$ axis by $45^{\circ}$ and, then, $\mathrm{CCW}$ around $\mathrm{Z}$ axis by $70^{\circ}$ (Figure 2).

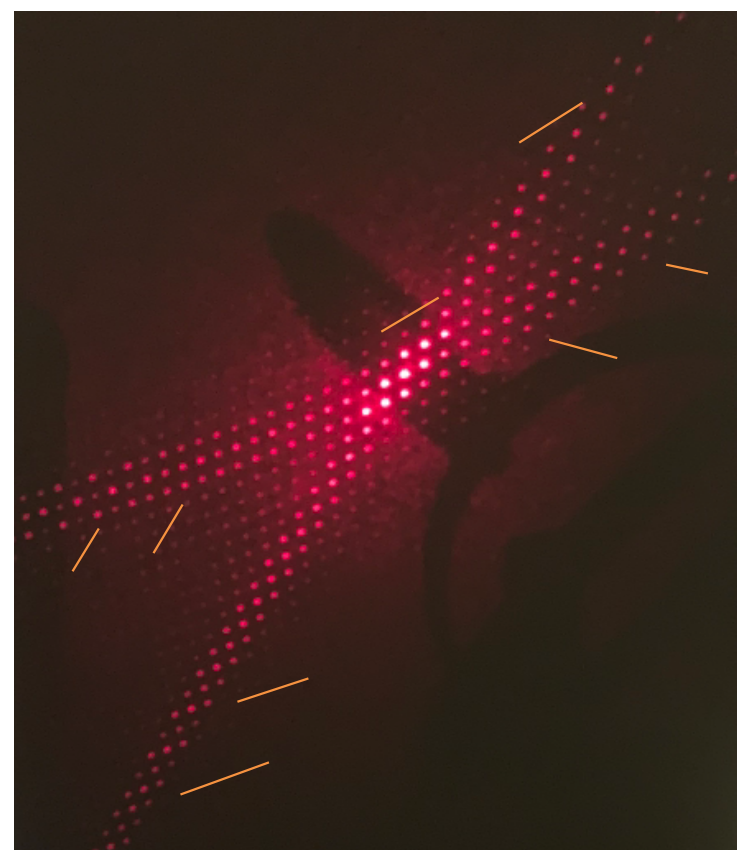

Figure 2. Patterns: rotating grating $\mathrm{CCW}$ around $\mathrm{X}$ by $45^{\circ}, \mathrm{CW}$ around $\mathrm{Y}$ axis by $45^{\circ}$ and $\mathrm{CCW}$ around $\mathrm{Z}$ axis by $70^{\circ}$

Observation: The comparison of Figure $03 \mathrm{~b}$ and Figure 2 shows that the third rotation (around the $\mathrm{Z}$ axis) causes that each branch of the patterns expanded more, curved more, and inclined more towards the horizontal axis. The pattern as a whole rotates CW.

Experiment-2 shows the novel phenomena that each branch of the pattern is expanded nonuniformly. More specifically, the expansion of the spacing between the adjacent diffraction orders of each branch of the pattern increase gradually from the bottom-portion to the top-portion of the pattern. The short orange lines show the distances between 5 diffraction orders on each branch of the pattern. 


\subsection{Rotating 2D-Cross-Grating CCW Around X Axis by $45^{\circ}, \mathrm{CCW}$ Around Y Axis by $45^{\circ}$, Then}

\section{Rotating $\mathrm{CW}$ and $\mathrm{CCW}$ Around $\mathrm{Z}$ axis}

Next, let us rotate the grating $\mathrm{CCW}$ around $\mathrm{X}$ axis by $45^{\circ}$ and $\mathrm{CCW}$ around $\mathrm{Y}$ axis by $40^{\circ}$ (Figure 04b). Figure 04a shows the pattern after the first rotation (around the $\mathrm{X}$ axis) of the apparatus. Figure 04b shows that the second rotation ( $\mathrm{CCW}$ around the $\mathrm{Y}$ axis) causes that each branch of the patterns expanded, curved, and inclined towards the vertical axis.

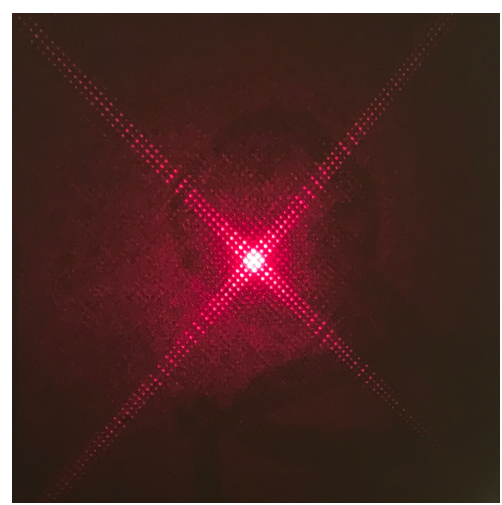

(a)

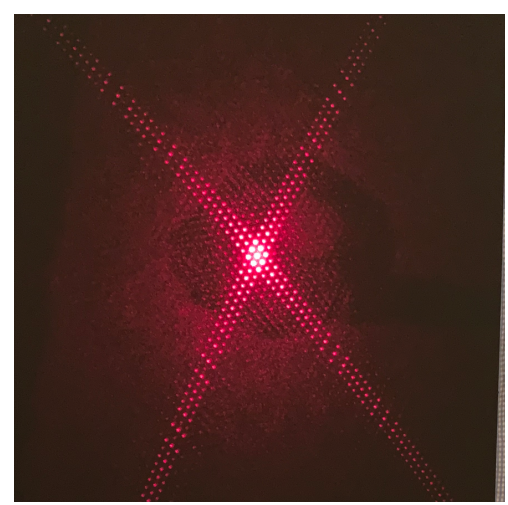

(b)

Figure 04. Patterns; (a) 2D-cross-grating rotating $\mathrm{CCW}$ around $\mathrm{X}$ axis by $45^{\circ}$; (b) 2D-cross-grating rotating $\mathrm{CCW}$ around $\mathrm{X}$ axis by $45^{\circ}$ and, then rotating $\mathrm{CCW}$ around $\mathrm{Y}$ axis by $40^{\circ}$

From the orientation of the 2D-cross-grating (Figure 04b), performing the following two Experiments, respectively: rotating the 2D-cross-grating $\mathrm{CW}$ and $\mathrm{CCW}$ around $\mathrm{Z}$ axis respectively. Experiment-3: Rotating the grating: CCW X $\left(45^{\circ}\right)+\mathrm{CCW} Y\left(40^{\circ}\right)+\mathrm{CW} \mathrm{Z}\left(70^{0}\right)$

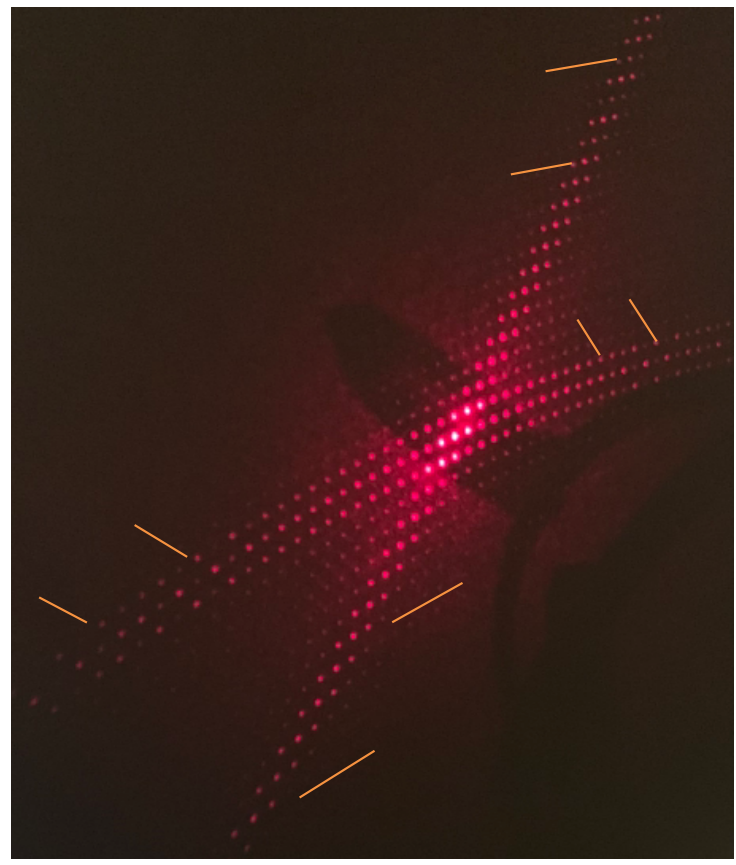

Figure 3. Patterns: rotating grating $\mathrm{CCW} 45^{\circ}$ around $\mathrm{X}$ by $45^{\circ}, \mathrm{CCW}$ around $\mathrm{Y}$ axis by $40^{\circ}$ and $\mathrm{CW}$ around $\mathrm{Z}$ axis $70^{\circ}$ 
Observation: The comparison of Figure $04 \mathrm{~b}$ and Figure 3 shows that the third rotation (around the $\mathrm{Z}$ axis) causes that each branch of the patterns expanded more, curved more, and inclined more towards the horizontal axis. The pattern as a whole rotates $\mathrm{CW}$.

Experiment-3 shows that the pattern is expanded non-uniformly (shown by the short orange lines on each branch of the pattern). Namely, the expansions of the spacings between the adjacent diffraction orders increase gradually from the top-portion to the bottom portion of each branch of the pattern.

Experiment-4: Rotating the grating: $\mathrm{CCW} X\left(4^{\circ}\right)+\mathrm{CCW} Y\left(40^{\circ}\right)+\mathrm{CCW} \mathrm{Z}\left(\mathbf{7 5}^{\circ}\right)$

Rotating the grating $\mathrm{CCW}$ around $\mathrm{X}$ axis by $45^{\circ}, \mathrm{CCW}$ around $\mathrm{Y}$ axis by $40^{\circ}$ and, then, $\mathrm{CCW}$ around $\mathrm{Z}$ axis by $70^{0}$ (Figure 4$)$.

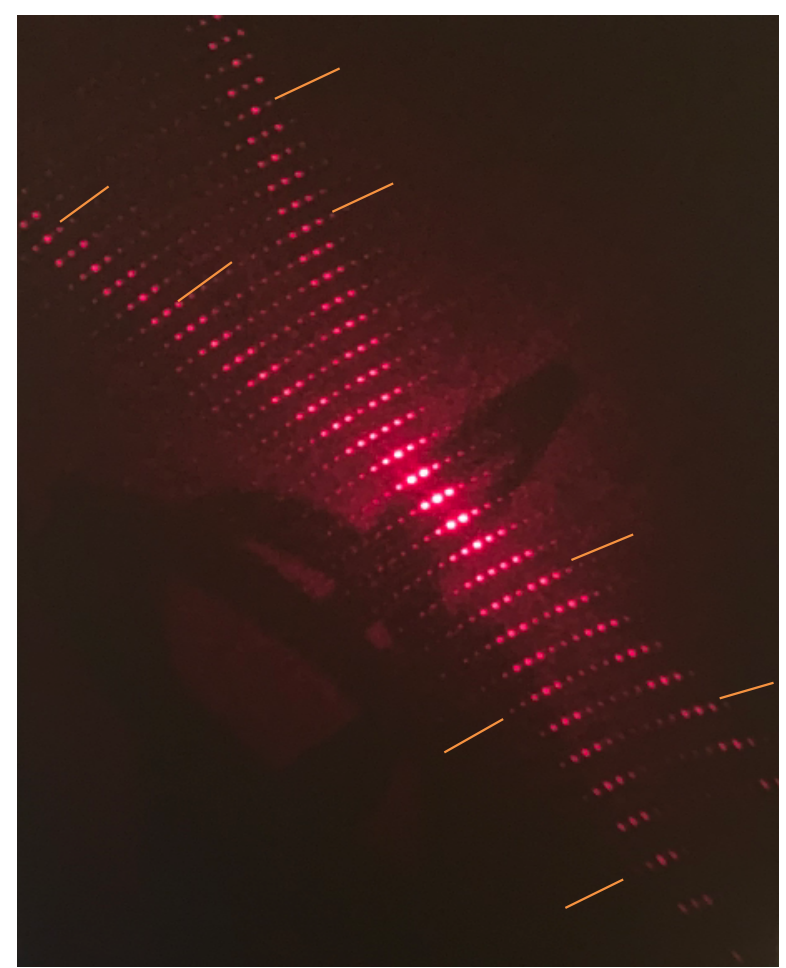

Figure 4. Patterns: rotating CCW grating around $\mathrm{X}$ by $45^{\circ}, \mathrm{CCW}$ around $\mathrm{Y}$ axis by $40^{\circ}$ and $\mathrm{CCW}$ around $\mathrm{Z}$ axis by $75^{\circ}$

Observation: The comparison of Figure $04 \mathrm{~b}$ and Figure 4 shows that the third rotation (around the $\mathrm{Z}$ axis) causes that each branch of the patterns expanded more, curved more, and inclined more towards the horizontal axis. The pattern as a whole rotates CW.

Experiment-4 shows that the pattern is expanded non-uniformly. Namely, the expansions of the spacing between the adjacent diffraction orders increase gradually from the top-portion to the bottomportion of the pattern.

\section{Discussion}

The preliminary experiments of this article show, the first time: 
(1) the non-uniform spacings between adjacent diffraction orders of the pattern of the 2D-cross-grating. More specifically, the spacings are gradually increase either from the top-portion to the bottom-portion of the pattern, or from the bottom-portion to the top-portion of the pattern.

(2) the phenomena of the non-uniform spacings exist regularly.

The phenomena challenge both the standard theory of the 1D-grating for the normal incident light beam and the theories of 1D-grating for the oblique incident light beam. For example, see [8-11] and references within.

\section{Appendix: Non-uniform Pattern of 1D-Grating [7]}

By rotating the 1-D-grating around three axes sequentially, we observed the non-uniformity of the diffraction patter (pictures below) [7].

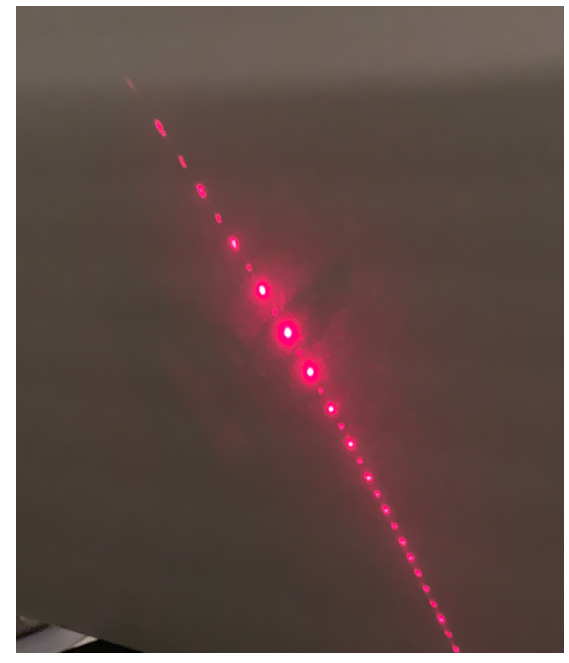

(a)

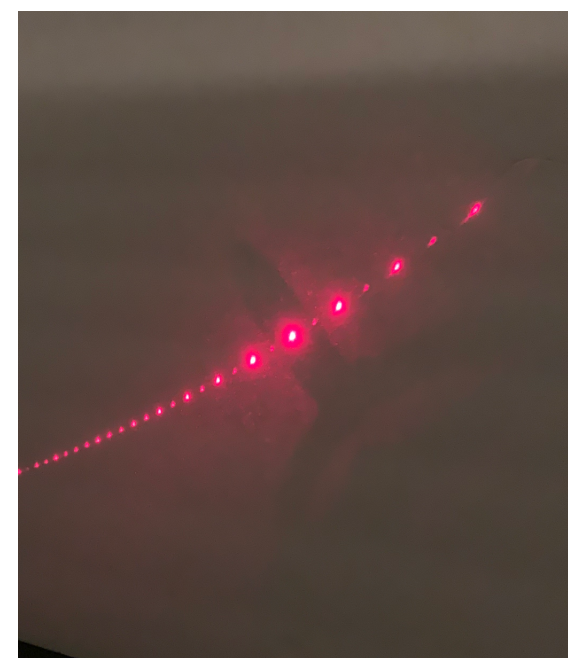

(c)

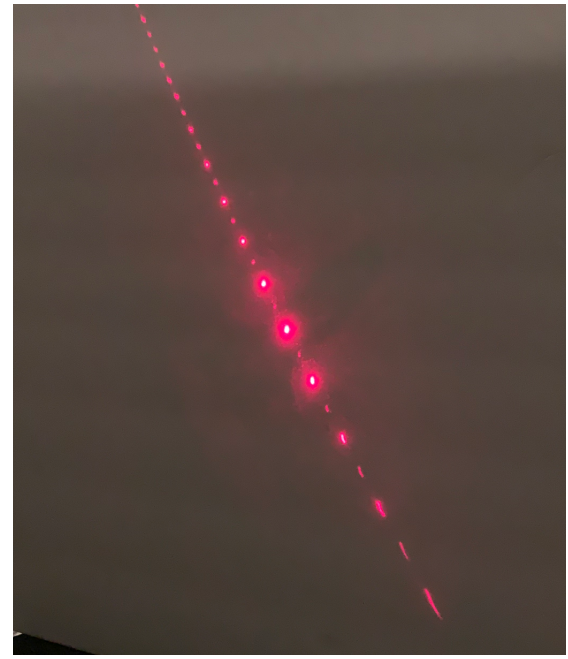

(b)

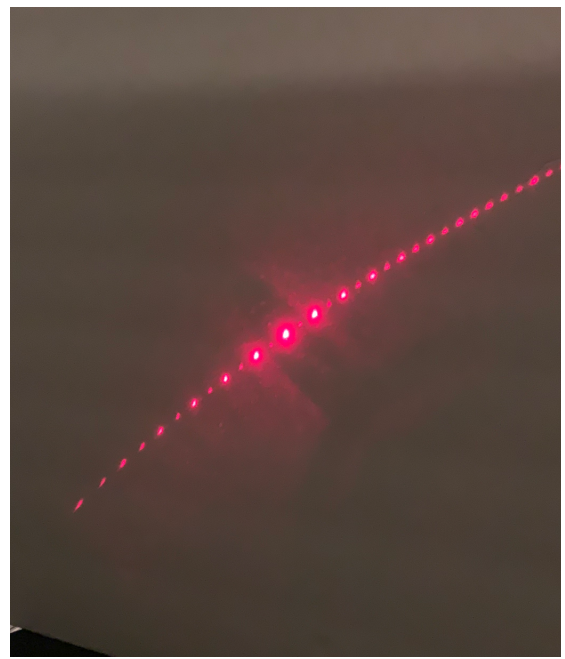

(d) 


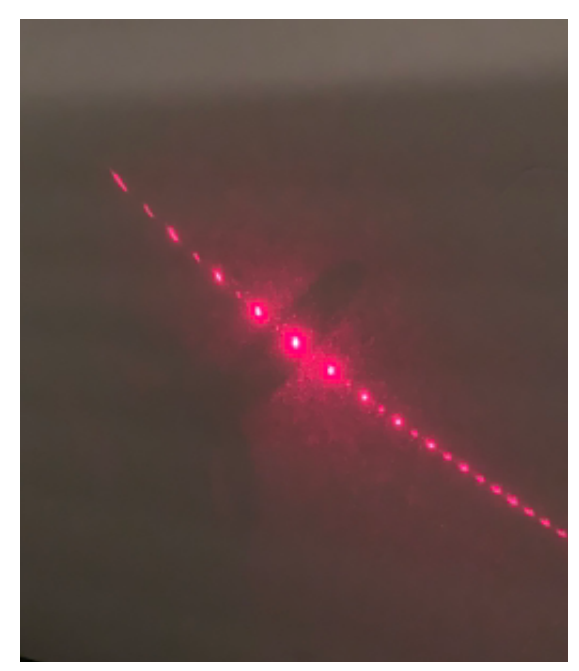

(e)

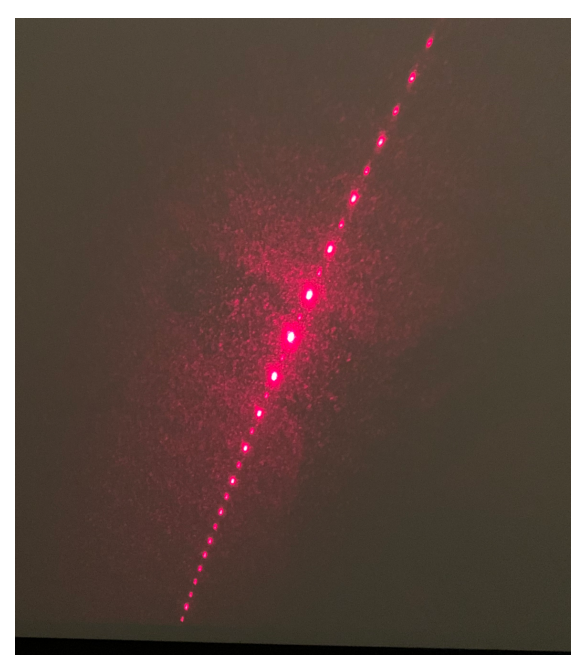

(g)

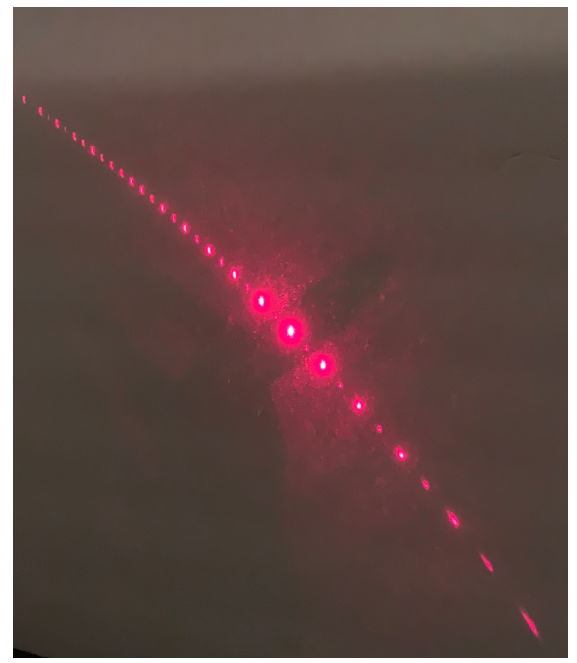

(f)

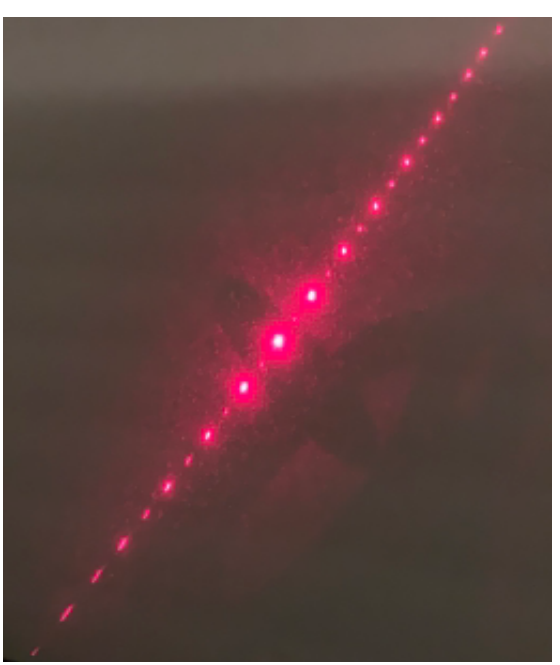

(h)

Figure A. Non-uniformity of Pattern of 1D-grating rotating around 3 axes sequentially

The comparison of the non-uniform spacings of the patterns of the 1D-grating (Appendix [7]) and of the 2D-cross-grating (Figure 1-4) shows that the spacing-increasing rate of the 1D-grating is larger than that of the 2D-cross-grating.

\section{References}

[1] Hui Peng, "Novel Double Slit and Cross-Double Slit Experiments--- Interference Patterns Depending on Orientation of Diaphragm”, Research Square, preprint, DOI: https://doi.org/10.21203/rs.3.rs-653201/v1, June 2021.

[2] Hui Peng, "Novel Mystery of Double Slit/Cross-double Slit Experiments--- Curved Interference Pattern and Interference Pattern Depending on Orientation of Diaphragm”, Research Square, preprint, DOI: https://doi.org/10.21203/rs.3.rs-701216/v1, July 2021.

[3] Hui Peng, "Novel Universal Phenomena of Single Slit, Double Slit, Cross-double Slit and Triple 
Slit Experiments --- Curved Patterns and Orientation-Dependence of Patterns", Research Square, preprint, DOI: https://doi.org/10.21203/rs.3.rs-722731/v1, July 2021.

[4] Hui Peng, "Curved, expanded and inclined patterns of dynamic double slit/cross-double slit/cross-grating experiments”, J. Eng. 1-20. https://doi.org/10.1049/tje2.12101, Nov. 2021.

[5] Hui Peng, “Curved, Expanded and Inclined Diffraction Patterns of Grating---Rotating Grating Around Three Axes (1)”, Research Square, preprint, DOI: https://doi.org/10.21203/rs.3.rs-1209507/v1, December 2021.

[6] Hui Peng, "Curved, Expanded, Inclined and Mirror-Symmetric Diffraction Patterns of 2D-Cross-Grating---Rotating Grating Around Three Axes (2)", Research Square, preprint, DOI: https://doi.org/10.21203/rs.3.rs-1244947/v1, January 2022.

[7] Hui Peng, "Non-Uniform, Curved, Expanded and Inclined Diffraction Pattern of 1D-Grating Experiments ---Rotating Grating Around Three Axes”. TechRxiv. Preprint. https://doi.org/10.36227/techrxiv.19134743.v1, February 2022.

[8] James E. Harvey and Cynthia L. Vernold, "Description of Diffraction Cross-grating Behavior in Direction Cosine Space”, Applied Optics 37(34):8158-8160. DOI: 10.1364/AO.37.008158, Dec. 1998.

[9] C. Braig, et al., “An EUV beam-splitter based on conical grazing incidence diffraction”, Optics Express, Vol. 20, No. 2 / 1825, January 2012.

[10] Ninad R. Jetty, Akash Suman and Rajesh B. Khaparde, "Novel cases of diffraction of light from a cross-grating: Theory and experiment”, American Journal of Physics 80(684):972.

DOI: $10.1119 / 1.4737854,2012$.

[11] Georg Heuberger, et al., "Light diffraction from a phase cross-grating at oblique incidence in the intermediate diffraction regime", Applied Physics B 127:72, https://doi.org/10.1007/s00340-021-07620-x, April 2021. 